\title{
Estrategia metodológica para la enseñanza- aprendizaje de las actividades deportivas mediante el uso de TIC
}

Methodological strategy for teaching-learning of sports activities through the use of ICT

Sergio Manuel Sepúlveda

Corporación Universitaria Minuto de Dios.

Correo electrónico: ssepulveda2@uniminuto.edu.co 


\section{Resumen}

En este artículo se presenta la estrategia metodológica (pedagógica) para la enseñanza aprendizaje de actividades deportivas, con el uso de las aulas virtuales y las Tecnologías de la información ya la comunicación (TIC). Para ello, desglosaremos todo lo que enmarca este conocimiento y entraremos a orientar el proceso, en el que se ha venido desarrollando nuestro estudio, para poder analizar la problemática que enmarca la falta de realización de actividades deportivas y físicas de estudiantes de educación superior a distancia. Para ello evaluaremos una estrategia metodológica, que contrarreste la problemática frente al desarrollo educativo de los estudiantes de esta modalidad (distancia).

Palabras clave: Aulas virtuales, TIC, actividades deportivas, educación superior.

\section{Abstract}

This article presents the methodological (pedagogical) strategy for teaching learning sports activities, with the use of virtual classrooms and information and communication technologies (ICT). To do this, we will break down everything that frames this knowledge and we will enter to guide the process, in which our study has been developed, in order to analyze the problem that frames the lack of performance of sports and physical activities of students of distance higher education. To do this, we will evaluate a methodological strategy that counteracts the problem against the educational development of students of this modality (distance).
Keywords: Virtual classrooms, ICT, sports activities, higher education.

\section{Introducción}

En Colombia se ha evidenciado el avance en las Tecnologías de la información y la comunicación (TIC), para el desarrollo investigativo, científico y sociocultural, ya que hoy en día la mayoría de sujetos está en la capacidad de manejar este tipo de herramientas, pero es necesario crear hábitos, desarrollar ideas prácticas para el uso de las mismas y buscar la implementación en cada una de las áreas donde se observa la tradicionalidad.

Todos los procesos hoy en día, de alguna manera, se ven impactados por el uso de la tecnología, es por ello que debemos buscar estructurar procesos educativos para el deporte (en cada disciplina), ya que esto nos permitirá el desarrollo de prácticas deportivas, y permitirá que los alumnos obtengan una herramienta de trabajo para su continuo mejoramiento, o puedan desarrollarlo el hábito deportivo con un objetivo determinado a partir de la introducción a la práctica.

Las posibilidades serán amplias porque nos ayudan a reforzar los estímulos que se brinden dentro de una clase, fomentaran la práctica deportiva en cada espacio donde se tenga el acceso y ayudaran a potenciar las habilidades deportivas en los sujetos con miras al aprendizaje en búsqueda de rendimiento eficaz y óptimo.

La viabilidad de la investigación, parte desde su implementación ya que se puede direc- 
cionar por medio de la plataforma que maneja el aula virtual, la idea presenta una factibilidad pensando en que se mejora la formación que se oferta para la población estudiantil que maneja la universidad, en lo económico es muy rentable porque su trabajo se desarrolla a partir de lo establecido por la institución.

El objetivo es fomentar la práctica deportiva desde el factor de desarrollo pedagógico, que es el propósito educativo que desarrolla la universidad; en el paso a paso vincularemos y promocionaremos los aspectos educativos de una formación integral y completa para cada estudiante y ofrecerle cobertura en el proceso de enseñanza y aprendizaje.

La construcción del proceso parte desde el desarrollo metodológico que se maneja en el deporte y la actividad física, ya que este genera un impacto en la formación de cada uno de los estudiantes a los cuales se les aplicará el proceso educativo.

\section{Ambientes de aprendizaje de tipo virtual encaminados al desarrollo de la actividad física y el deporte}

Para el desarrollo de la actividad física y el deporte en la actualidad, se ha convertido en un verdadero reto el aplicar las herramientas de tipo tecnológico (TIC) en pro de su desarrollo de manera que esto sea convertido en una herramienta en los procesos de formación que se derivan de todas las acciones motrices y que se generan desde lo práctico, ya que se entiende que desde que las experiencias de formación, en esta área, deben estar acom- pañadas de experiencias de lo que vivencian dentro del escenario, pero también se debe resaltar la importancia de la formación desde lo teórico como los elementos de autoaprendizaje como plantean Foguet \& Donés (2011) “Estas experiencias se basan no sólo en un cambio de escenario para pretender cambiar el aula, que para nosotros es el gimnasio o la pista polideportiva, por un espacio virtual intangible; proponen nuevos métodos y modalidades para organizar, desarrollar y evaluar los aprendizajes a partir de un modelo pedagógico centrado en las competencias y en la evaluación formativa", ya que encontramos que las herramientas de tipo virtual juegan un papel importante en el desarrollo de los procesos de enseñanza y aprendizaje del deporte, la recreación y educación física.

Es claro entender que este tipo de formación cambia el paradigma que desde lo tradicional se caracterizaba, porque desde la concepción del deporte, la recreación y actividad física se entiende que el proceso se realiza únicamente desde lo presencial y lo práctico en el campo de acción, que todas las características que se emplean en su capacitación se brindan desde su aplicación en el escenario en que el estudiante lo ejecuta, con nociones que brinda el entrenador o profesor, pero en la actualidad este paradigma se ha roto por las diferentes estrategias de las herramientas tecnológicas y de comunicación que se han aplicado para beneficio de las ciencias del deporte.

Para ello se debe contemplar el modelo de formación virtual, con ayuda de las herramientas tecnológicas y de la comunicación, en áreas de desarrollo del deporte, la recreación y actividad física, como una alternativa metodológica con el objetivo de estructurar un estilo 
de enseñanza y aprendizaje innovador de calidad en un escenario o ambiente de aprendizaje alterno al tradicional.

\section{Orientación de competencias deportivas por medio de herramientas virtuales}

Durante el desarrollo deportivo se evidencia que gráficamente se puede desarrollar un aprendizaje en aspectos técnicos, físicos, tácticos y psicológicos, todo esto se aporta de una manera teórica donde se ilustra por medio de video, graficas, textos etc. donde podemos decir que el alumno aprende visualizando y realizando repeticiones continuas, que le enmarquen tareas donde le involucren la ejecución, con una conciencia de lo que está realizando, para que la pueda emplear en diferentes situaciones, que en este caso las llamaremos como "situaciones reales de juego", que es donde el realiza la toma de decisiones.

Analizando lo que se plantea, se evidencia la utilidad de la utilización de una herramienta virtual para la enseñanza y aprendizaje, para lo cual la academia debe plantear:

- Una estructura curricular que brinde la posibilidad de enlazar o articular el contexto teórico con el contexto práctico dentro de un currículo con diseño que contemple la didáctica y la lúdica de lo que se enseña.

La formación que se brinda desde el aula virtual, debe garantizar trabajos que complementen el desarrollo de cada una de las dimensiones que la institución educativa considere conveniente; su estructura debe manejar una estructura transversal, que permita la formación de un profesional, que desde la plataforma de enseñanza, pueda tener los elementos pedagógicos suficientes dentro de su preparación en el área de competencia.

Este modelo de educación nos permite evidenciar el desarrollo deportivo de los estudiantes universitarios, por medio de una herramienta tecnológica, en la que su orientación está determinada de una manera académica, pensada pedagógicamente para dar una respuesta positiva de obtención de conocimiento. Según lo que nos plantea Castillo y Álvarez (2013), el modelo de "enseñanza utilizado para la asignatura de estructura y organización de Instituciones Deportivas fue evaluado, teniendo en cuenta las incidencias que pudieran ocurrir en dicha plataforma", para ello debemos analizar que el modelo puede tener la misma funcionalidad en el contexto que se aplica, y para la cual tiene como objetivo de enseñanza y aprendizaje. Castillo y Álvarez nos brindan la intencionalidad para la aplicación de las TIC en la asignatura dedicada a los estudiantes, y como se desarrolla ese complemento: "por tanto, la evaluación de la plataforma WebCT como recurso didáctico para la asignatura de Estructura y Organización de Instituciones Deportivas ha sido muy positiva, recomendando su uso como apoyo tanto al docente como al discente en su proceso de enseñanza- aprendizaje"(2013).

Siguiendo lo anterior, es importante abordar la temática del proceso educativo que se realiza con herramientas tecnológicas en el deporte, porque ellas permiten evidenciar el proceso metodológico que realiza el docente o entrenador para multiplicar el conocimien- 
to de una manera que el estudiante entienda, analice y ejecute lo que se le plantea, es decir pueda ser un promotor del aprendizaje pedagógico recibido.

Todos los aprendizajes, por medio de herramientas virtuales y tecnológicas, evidencian un impacto positivo frente al proceso de enseñanza transmitido al individuo, porque le aporta como un medio de aprendizaje que le ayuda a orientarse y capacitarse, permitiéndole una formación teórica para su desarrollo práctico, o, en diferentes casos, que promueva el conocimiento a través del análisis que la información tecnológica le trasmite, esto es importante porque lo ilustra gráficamente y se presenta de una forma lúdica y didáctica, que facilita la obtención del conocimiento.

Estos mecanismos de enseñanza, en particular para el área deportiva, son muy funcionales, porque se ha determinado que no es solo generar un conocimiento en el estudiante de manera gráfica, sino que lo aplique de una manera analítica y consiente para su desarrollo motriz, y en concordancia con la tarea específica a la que se debe aplicar.

Es importante lo que plantean Chacón Cuberos et al, (2016, p.114-115), ya que evidencian la enseñanza que brindan los videojuegos en el área deportiva de la siguiente manera "Los procesos educativos han sufrido un cambio radical en las últimas décadas, abarcando una profunda transformación, llegados a este punto, conviene destacar que los centros educativos españoles han sufrido una fuerte inmersión de las tecnologías digitales". Por su parte, López (2014), establece los objetivos de esta modalidad de educación virtual, la cual debe garantizar bajo la estructura curricular "lograr el desarrollo de habilidades cognitivas y comunicacionales es una de las principales metas que persigue la educación actual, siendo el objetivo principal la consecución de un aprendizaje significativo e integral, marcado por los componentes de experiencias y social".

Los estudios han evidenciado que el aprendizaje, a partir de tecnologías, es un buen mecanismo para el desarrollo de las capacidades de los estudiantes, que las herramientas de uso tecnológico son una buena alternativa para desarrollar capacidades que desde lo visual pueden tener una formación, que a partir de cosas muy simples como el realizar una actividad virtual se puede obtener un gran conocimiento en lo físico y cognitivo, que es de gran relevancia para el desarrollo de sus capacidades, como plantea López (2014): “que además, se valga de una gran riqueza metodológica e instrumental, determinan algunas de las múltiples ventajas que supone la utilización de las TIC en los procesos de enseñanza-aprendizaje escolar, como son el aumento y mejora de la oferta informativa, la eliminación de barreras espacio-temporales, la facilitación de trabajo colaborativo o el aumento de la flexibilidad del aprendizaje; cristalizando todas ellas, en la demanda de métodos y recursos que se estableció con anterioridad para alcanzar un aprendizaje total".

Este estudio en particular evidencia los procesos de enseñanza que se generan a partir de la utilización de elementos tecnológicos que son de gran relevancia hoy en día, por eso este tipo de formación debe cumplir unas características que forman en sus diferentes dimensiones al sujeto que hace uso de ellas.

En definitiva, este estudio lo que pretende es analizar la actitud de los escolares frente a 
los procesos virtuales, en el proceso formativo apoyado en esta tecnología. López (2014), se refiriere al "trabajo virtual como instrumento o material en determinadas sesiones de educación física, constituyendo estos un recurso extraordinario para evitar la monotonía en el área, a la vez que eficaz en la consecución de objetivos educativos generales y específicos".

Como lo planteamos anteriormente, como estudio de proceso de enseñanza- aprendizaje frente a una actividad de tipo virtual, se deben contemplar los objetivos que plantea López (2014):

“Determinar y describir parámetros de ocio-digital, actividad física y experiencias previas con videojuegos activos en una población de alumnado de educación primaria".

“Analizar la relación existente entre la posibilidad de uso de exergames en educación física con el género, los índices de actividad física y los parámetros de ocio-digital".

Lo que nos brinda este trabajo metodológico está orientado hacia el proceso educativo que se estructura a través de la herramienta virtual (para este caso los video-juegos), y de cómo a partir una actividad de interés propio se generan aprendizajes de manera significativa en el individuo que la ejecuta.

Este planteamiento muestra que las herramientas tecnológicas son importantes para que aporten al desarrollo de la educación, en este caso en el área de la actividad física y el deporte. Este conocimiento debe estar en continuo complemento para que el proceso de enseñanza y aprendizaje aporte de manera didáctica y lúdica, donde el estudiante fortalezca sus capacidades de forma eficiente a partir del conocimiento que se le aportan, esto es de suma importancia para el rol que ejerce el docente o entrenador frente a su alumno o deportista, porque en él se puede realizar un proceso de análisis frente a sus fortalezas y aspectos a mejorar, así de igual manera apropie su aprendizaje como una herramienta que le ayuda en situaciones reales en el campo de ejecución.

Corredor Castro (2013, p.171-173), expone la enseñanza y aprendizaje de la educación física de la siguiente manera "un ambiente virtual de aprendizaje es el espacio físico donde las nuevas tecnologías materializadas en los sistemas satelitales, el internet, los multimediales, se han potenciado traspasando al entorno escolar, favoreciendo procesos pedagógicos comunicacionales, experiencias y nuevos espacios de aprendizaje. Los ambientes virtuales surgen con la finalidad de complementar, apoyar y reforzar la forma de enseñanza", basado en la potenciación de habilidades que se fortalecen en el ambiente escolar.

Los procesos de formación en los programas de educación física, deben asumir un liderazgo intelectual para ser un complemento de las demás asignaturas, a partir de la formación de experiencias pedagógicas donde el currículo se diseñe con una planeación y diseño adecuados, y que tengan aplicación en ambientes virtuales de aprendizaje, en que los estudiantes los puedan trasladar a las prácticas físico-deportivas.

Todas las estrategias pedagógicas deben estar direccionadas por el currículo, pero de- 
ben tener un objetivo por parte de la academia, pero en últimas quien orienta el proceso de innovación es el docente y quien le da la aplicabilidad es el estudiante, para que todo sea un complemento en su formación: el proceso educativo debe ser una experiencia que contribuya a la construcción de un individuo integral y que esta fortalecido en el uso de herramientas virtuales que son de utilidad para la preparación profesional, como nos ayuda a complementar en su exposición Corredor (2013): "en esta misma perspectiva, se propone un modelo pedagógico para los docentes de la acción motriz, cuyo fin es la utilización de las TIC y la web2.0 como una alternativa que permite responder a las necesidades de la 'era virtual', lo que exige a estudiantes y docentes, asumir retos en diálogos de enseñanza-aprendizaje con un modelo pedagógico: centrado en un estudiante flexible, colaborativo, interactivo y autónomo".

Todas las herramientas tecnológicas cumplen un papel fundamental en el desarrollo pedagógico que se tiene frente al desarrollo educativo de los estudiantes, dando cuenta de cómo se realiza un aprendizaje respecto a lo que se ilustra, por ende, este estudio es relevante para un objetivo de aprendizaje a nivel deportivo, porque a través del conocimiento que brinda la experiencia del juego podemos evaluar un aprendizaje respecto a las orientaciones que nos brinda el deporte como su parte técnica, su objetivo y la parte reglamentaria, es un claro ejemplo de cómo se da el aprendizaje a partir de su ejecución según lo que nos plantea en su tesis Corredor Castro (2013), ya que tiene como objetivo demostrar cómo una herramienta como "los videojuegos y la técnica del feedback extrínseco son una herramienta pedagógica que fortalece y faci- lita la comprensión de los reglamentos de baloncesto y tenis de campo".

Tal como lo plantea Sangra (2001), la educación y la virtualidad tienen que estar en comunión para la construcción del conocimiento, de tal forma que el fortalecer este vínculo podrá ser un mecanismo metodológico de formación de una manera óptima "No podemos hacer lo mismo en medios distintos, aunque nuestras finalidades educativas y, por tanto, los resultados que perseguimos sean los mismos, pero debemos saber de antemano que el camino que debemos recorrer es distinto".

El rol del docente en este planteamiento, le permite ser un formador que potencie el aprendizaje de sus estudiantes en el área deportiva, y, su papel le permite generar capacidades pedagógicas que fortalecen los mecanismos de enseñanza, haciendo un paralelo en lo tradicional, por ende, Corredor (2005), expone sus reflexiones: "Se considera que para los educadores físicos ha llegado el momento de incorporar y utilizar éstas nuevas tecnologías en su quehacer pedagógico como parte de la estrategia metodológica para ampliar, transferir, mejorar, crear o transformar los conocimientos que la disciplina deportiva debe impartir para mejorar su aprendizaje".

Lo anterior se complementa con el manejo que se presenta actualmente por parte del rol de los docentes, que son los que participan de manera activa en el proceso de enseñanza en el aprendizaje de los estudiantes "necesitamos hacer uso de estrategias y metodologías que respondan a las necesidades que actualmente demandan nuestros estudiantes. Dichas estrategias están referidas hacia el uso de nuevas tecnologías que permitan organizar ambientes 
de aprendizaje significativos, dinamizando de antemano las estrategias de enseñanza".

Es importante plantear que en la actualidad podemos desarrollar una unidad didáctica o una unidad de entrenamiento, con la ayuda de las plataformas tecnológicas, por ejemplo un aula virtual, pero lo más importante es la promoción de un modelo educativo que permita al estudiante aprender, y que lo haga de una manera que le desarrolle su conocimiento de una manera teórico-práctica en esta área, que al desarrollar su proceso educativo cumpla con los objetivos planteados y el desarrollo de sus capacidades como lo indica Castro (2013): "la virtualidad se utilizaría para apoyar, complementar o reforzar didácticamente los procesos y acciones de cualquier práctica motriz como medio para estimular el aprendizaje significativo. Con la interactividad, el estudiante puede pedir información, animarse a ingresar en temas nuevos cuando tenga aprendidos los anteriores y así lograr sus intereses personales.

\section{Estrategia metodológica}

Las nuevas tendencias en las tecnologías de la información y la comunicación, y su desarro1lo, generan un impacto en la gestión educativa frente al progreso de la sociedad, y han permitido transformar los sistemas de enseñanza de los diferentes ambientes de aprendizaje, tanto presenciales y a distancia, de tal forma que hoy no se puede calcular la dimensión de información y su acceso es casi ilimitado, pues cuenta con una accesibilidad para cada una de las personas en las diferentes áreas de conocimiento.
Estos elementos didácticos desarrollan capacidades pedagógicas de orden educativo, y presentan un impacto positivo para la formación de los futuros profesionales de los diferentes sectores o áreas de conocimiento. Al hablar de la formación profesional de tipo virtual, entendemos que se ha convertido en una herramienta didáctica que ayuda a la sociedad a formar profesionales con calidad, con la ayuda de una metodología diferente a la convencional. El modelo es innovador en la actualidad, porque maneja diferentes herramientas como el aula virtual de aprendizaje, por otra parte, la creación de esta estrategia o propuesta educativa, además de integrar las TIC al medio deportivo, permite trasmitir diferentes conceptos y contenidos como base fundamental en la creación del conocimiento. Adicional a lo anterior, promueve los espacios de desarrollo técnico y de conocimiento deportivo, estos interpretados aquí como espacios y ambientes que son extensiones para la práctica y formación integral en la dimensión física, ya que este proceso de enseñanza aprendizaje tiene un planteamiento acerca de cambiar el paradigma de formación que se tiene en el área deportiva.

\section{Objetivo general}

Proponer una estrategia virtual para la formación deportiva de los estudiantes de la Corporación Universitaria Minuto de Dios. 


\section{Objetivo específicos}

- Diseñar un aula virtual que promueva la formación deportiva en los estudiantes.

- Evaluar los elementos de formación deportiva que promueven la estrategia con ayuda de las TIC.

La estrategia de formación deportiva con ayuda de las TIC es una reflexión tecnológica y pedagógica para el desarrollo de una acción didáctica, con nuevas formas de entender el aprendizaje y que influye en el desarrollo del área de conocimiento del deporte y de la actividad física, a partir de lo planteado de otras relaciones de los espacios y los tiempos, en pos de la realización de una acción formativa pensada, desarrollada y estructurada para los estudiantes que realizan su proceso de formación bajo la metodología de distancia tradicional.

Promover en las prácticas docentes nuevas herramientas de enseñanza apoyadas en las TIC, ayudará a la formación integral en el área física y cognitiva del profesional en formación, por medio de la plataforma que maneja las aulas virtuales de las diferentes materias y de los contenidos del currículo de cada pregrado. Sin embargo, Rodríguez (2010) aduce "que uno de los principales objetivos es que son pensados en las necesidades de los estudiantes o la institución, ya que solo se involucra una dimensión pedagógica y operacional tecnológica".

Siguiendo lo anterior, desde la Universidad podemos fortalecer todos los procesos de enseñanza-aprendizaje para la formación integral del estudiante, y garantizar su desarrollo como futuro profesional, por lo cual se debe realizar un proceso de acompañamiento continuo desde su aula virtual y con maestros que faciliten, deleguen y retroalimenten la estrategia que se implementa para tener estudiantes con una formación integral idónea de manera que sea participativo y autónomo en el desarrollo teórico-práctico de su conocimiento.

\section{Conclusiones}

Se estructuró una estrategia metodológica pensada como una alternativa para la enseñanza y aprendizaje de actividades deportivas, para la formación de competencias en la dimensión física, a lo cual la Universidad le apunta con el objetivo de formar un profesional integral, y con la base sólida que se direcciona frente a los intereses-necesidades que se detectaron en los estudiantes del Centro Regional Soacha.

Al plantear una estrategia metodológica, para la enseñanza-aprendizaje de actividades deportivas mediante el uso de TIC, se encontró que efectivamente los estudiantes tienen gran afinidad para el desarrollo deportivo mediante actividades de tipo virtual, y se evidenciaron que se presenta congruencia frente a su proceso de aprendizaje y al fortalecimiento de formación en la dimensión física, ya que responden a los planteamientos teóricos que derivan de los aspectos metodológicos con los cuales se estructuró la propuesta, entendiendo que los estudiantes tienen afinidad con la metodología virtual y el desarrollo de actividades que son de interés para su formación.

Para los estudiantes de la Corporación Universitaria Minuto de Dios, Centro Regional 
Soacha, en la modalidad distancia tradicional, fue innovador frente a su desarrollo como profesional, en su proceso de formación frente a las competencias en las diferentes dimensiones, pero particularmente en el área física, pensando en que había que mejorar según la visión entregada, por medio de la estrategia utilizada por medio de la formación físico-deportiva en las aulas virtuales.

De acuerdo a esto se determinó que la estrategia desarrolla aspectos relevantes en los estudiantes del Centro Regional Soacha, las cuales destacamos frente al desarrollo deportivo y la promoción de hábitos de vida saludable bajo la orientación pedagógica de la metodología de formación en la cual estudian, por lo cual podemos decir que el proceso de enseñanza y aprendizaje es efectivo en la medida que se oriente frente a las necesidades e intereses de los estudiantes en procura de su desarrollo profesional de forma integral.

\section{Referencias}

Acuña Beltrán, L.F. (2016). Ambientes de aprendizaje: espacios, interacciones y mediaciones para construir saberes. Magazín Aula Urbana, 102. Recuperado de: https:// revistas.idep.edu.co/index.php/mau/article/view/956

Alfonso Sánchez, I. (2003). La educación a distancia. Acimed, 11(1). Recuperado de: http://scielo.sld.cu/scielo.php?script=sci_ arttext\&pid=S1024- 94352003000100002

Altea (1999). La salud mental mejora con el deporte. Centro de Psicología Altea. Recu- perado de: http://www.centroaltea.com/ la-salud-mental-mejora-con-el-deporte/

Castillo-Rodríguez, A. \& Álvarez-Kurogi, L. (2013). Aplicación de las TIC en estudiantes de ciencias de la actividad física y del deporte: plataforma virtual WebCT. Historia y Comunicación Social, 18, 279-290. Recuperado de: https://revistas.ucm.es/index. php/HICS/article/viewFile/44328/41887 DOI: https://doi.org/10.5209/rev_ HICS.2013.v18.44328

Aristizábal, J. F. (2005). Beneficios de la actividad física en la enfermedad cardiovascular. Archivos de Medicina, 10, 34-41. Recuperado de: http://www.redalyc.org/ pdf/2738/273820325009.pdf

Barberà, E. \& Badia, A. (2005). El uso educativo de las aulas virtuales emergentes en la educación superior. Universidad y Sociedad del Conocimiento. Recuperado de: http:// campusmoodle.proed.unc.edu.ar/file. php/513/Biblioteca/El_uso_educ_de_las_ aulas_vi rtuales_emergentes_en_la_educ_ supE_Barbera.pdf

Braun, H. (1980). Pedagogía educación y formación en el deporte. Educación Física y Deporte, 2(2). Recuperado de: http:// aprendeenlinea.udea.edu.co/revistas/index.php/educacionfisicaydeporte/article/ view/11188/10260

Chacón Cuberos, R., Castro Sánchez, M., Zurita Ortega, F., Espejo Garcés, T. \& Martínez Martínez, A. (2016). Videojuegos activos como recurso TIC en el aula de Educación Física: estudio a partir de parámetros de ocio digital. Digital Education Review. Re- 
cuperado de: http://www.raco.cat/index. php/DER/article/view/312491

Coll, C. (2008). Aprender y enseñar con las TIC: expectativas, realidad y potencialidades. Boletín de la Institución Libre de Enseñanza, 72. Recuperado de: https://www. educ.ar/recursos/70819/aprender-y-ensenar-con-las-tic-expectativas-realidad-y-potencialidades

Corredor, S. (2013). Uso de herramientas web en la educación física. Revista Corporeizando. Recuperado de: https://revistas.pedagogica. edu.co/index.php/corporeizando

El Espectador (2010). Coldeportes presentó su nuevo programa escuelas virtuales. Recuperado de: http://www.elespectador.com/ deportes/coldeportes-presento-su-nuevoprograma-escuelas-virtual-articulo-203126

González, A., Calleja, V., López, L., Padrino, P. \& Puebla, P. (2009-10). Los estudios de encuesta. UAM. Recuperado de: https://docplayer. es/12170823-Los-estudios-de-encuesta.html

González, C. \& Río, J. F. (2003). La enseñanza del deporte desde una metodología cooperativa. Tándem: Didáctica de la educación física, 10, 93100.

Hernández-Sampieri, R., Fernández-Collado, C. \& Baptista-Lucio, P. (2014). Metodología de la investigación. México: McGraw-Hill.

Londoño Orozco, J. (2010). La virtualidad en educación superior: una perspectiva. Recuperado de: http://www.virtualeduca.info/ ponencias2013/171/LavirtualidadenESUnaperspectivaJairoL.pdf
Martínez Rodríguez, J. (2011). Métodos de investigación cualitativa. Revista de la Corporación Internacional para el Desarrollo Educativo. Recuperado de: http://www.cide.edu. co/doc/investigacion/3.\%20 metodos $\% 20$ de\%20investigacion.pdf

Mayan, M .J. (2001). Una introducción a los métodos cualitativos: módulo de entrenamiento para estudiantes y profesionales. Recuperado de: https://sites.ualberta. $\mathrm{ca} /$ iiqm/pdfs/introduccion.pdf

Ministerio de Educación Nacional \& Coldeportes. (2010). Escuela Virtual de Deportes. Bogotá. Recuperado de: http://www. colombiaaprende.edu.co/html/productos/1685/w3-article-266801.html

Moreira-Segura, C. \& Delgadillo-Espinoza, B. (2015). La virtualidad en los procesos educativos: reflexiones teóricas sobre su implementación. Tecnología en Marcha, 28(1), 121-129. Recuperado de: http://www. scielo.sa.cr/pdf/tem/v28n1/0379-3982tem-28-01-00121.pdf

Organización Mundial de la Salud. (s.f.). Estrategia mundial sobre régimen alimentario, actividad física y salud. Recuperado de: http://www.who.int/dietphysicalactivity/ pa/es/

Pérez Ugidos, G., Laíño, A., Zelarayán, F. \& Márquez, S. (2014). Actividad física y hábitos de salud en estudiantes universitarios argentinos. Nutrición Hospitalaria, 30(4), 896-904.

Prieto, P. A. (2016). Guía de apoyo para el uso de Moodle. Universidad Minuto de Dios. 
Recuperado de: http://aulas.uniminuto. edu/inicio/recursos/Manual_Estudiantes. pdf

Ramírez Alarcón, L. S. (2013). El deporte como medio de formación integral. Tesis de grado. Maestría en sociología, Universidad del Valle: Cali. Recuperado de: http:// bibliotecadigital.univalle.edu.co/bitstream/10893/5702/1/0461897-p.pdf

Robles, J., Abad, M. \& Giménez, F. (2009). Concepto, características, orientaciones y clasificaciones del deporte actual. Efdeportes, 14(138). Recuperado de: http://www.efdeportes.com/efd138/concepto-y-clasificaciones-del-deporte-actual.htm

Rodríguez Sabiote, C., Lorenzo Quiles, O. Y Herrera Torres, L. (2005). Teoría y práctica del análisis de datos cualitativos. 2005. Proceso general y criterios de calidad. Revista Internacional de Ciencias Sociales y Humanidades, Sociotam, XV(2), Recuperado de: http://www.redalyc.org/ pdf/654/65415209.pdf

Sangrà, A. (2001). Enseñar y aprender en la virtualidad. Barcelona: Universidad Abierta de Catalunya.

Sarduy Domínguez, Y. (2006). El análisis de información y las investigaciones cuantitativa y cualitativa. Revista Cubana de Salud Pública, 33(3). Recuperado de: http://scielo. sld.cu/scielo.php?script=sci_arttext\&pi$\mathrm{d}=$ S0864-34662007000300020 